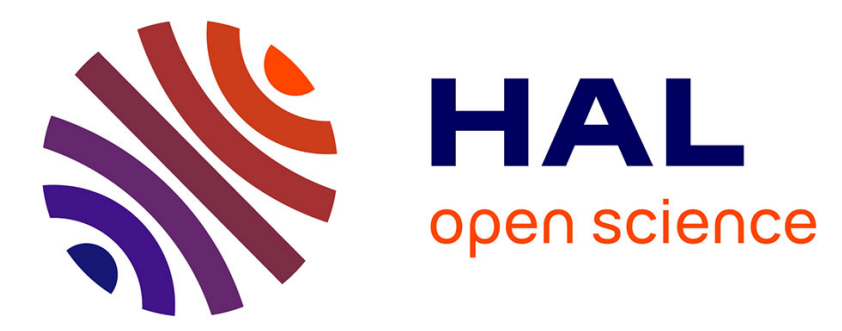

\title{
GeV Gamma-Ray Emission from Normal and Starburst Galaxies
}

Jürgen Knödlseder

\section{To cite this version:}

Jürgen Knödlseder. GeV Gamma-Ray Emission from Normal and Starburst Galaxies. Cosmic Rays in Star-Forming Environments, 34, pp.169 - 191, 2013, Astrophysics and Space Science Proceedings, 978-3-642-35409-0. 10.1007/978-3-642-35410-6_13 . hal-01381968

\section{HAL Id: hal-01381968 \\ https://hal.science/hal-01381968}

Submitted on 14 Oct 2016

HAL is a multi-disciplinary open access archive for the deposit and dissemination of scientific research documents, whether they are published or not. The documents may come from teaching and research institutions in France or abroad, or from public or private research centers.
L'archive ouverte pluridisciplinaire HAL, est destinée au dépôt et à la diffusion de documents scientifiques de niveau recherche, publiés ou non, émanant des établissements d'enseignement et de recherche français ou étrangers, des laboratoires publics ou privés. 


\title{
GeV gamma-ray emission from normal and starburst galaxies
}

Jürgen Knödlseder

\begin{abstract}
With the launch of the Fermi satellite and the advent of modern airCherenkov telescopes such as H.E.S.S. and VERITAS, star-forming galaxies have become a new class of gamma-ray sources that extend the study of cosmic-ray physics from the Milky Way to external galaxies. So far, 8 star-forming galaxies have been observed at GeV energies (SMC, LMC, Milky Way, M 31, M 82, NGC 253, NGC 1068, and NGC 4945) while 2 have been seen at TeV energies (M 82, NGC 253). In this paper, I review the observational status of the GeV observation of these galaxies, and I summarize the conclusions that have been drawn from these observations on cosmic-ray physics in normal and starburst galaxies.
\end{abstract}

\section{Introduction}

The Earth is permanently bombarded by a flux of charged particles, being primarily composed of atomic nuclei. The composition of the atomic nuclei follows closely that of the solar system matter, with a few notable exceptions, such as the overabundance of $\mathrm{Li}, \mathrm{Be}$ and $\mathrm{B}$, and other elements lighter than Fe. Electrons occur only with an abundance of about $1 \%$ in the particle flux, positrons are ten times less frequent.

This high-altitude radiation, discovered by Victor Hess in a series of balloon flights one century ago [1], is today known as cosmic rays. As we will demonstrate later, we know that these cosmic rays are ubiquitous in our Galaxy. The typical energy density of Galactic cosmic rays amounts to $\sim 1 \mathrm{eV} / \mathrm{cm}^{3}$ which is comparable to the energy density of starlight, of interstellar magnetic fields, and of the kinetic energy density of interstellar gas. Cosmic rays are thus an energetically important constitutent of our Galaxy that affect the physical state of the interstellar medium (ISM) by heating and ionizing its atomic and molecular phases [2]. Cosmic rays also

Jürgen Knödlseder

IRAP, 9, avenue Colonel-Roche, 31028 Toulouse Cedex 4, France, e-mail: jurgen.knodlseder@irap.omp.eu 
contribute to supporting interstellar gas against gravity [3], and are thought to alter the spectrum of interstellar turbulence as they get reaccelerated by draining energy from magnetohydrodynamic waves [4]. In addition, cosmic rays are essential for the Parker dynamo mechanism, which may be the source of the large-scale magnetic fields in galaxies like the Milky Way [5, 6].

The cosmic rays that are observed at Earth span an enormous energy range from about $10^{7} \mathrm{eV}$ to above $10^{20} \mathrm{eV}$ per nuclei. At the lowest energies $(\lesssim 10 \mathrm{GeV}$ per nuclei), cosmic rays are affected by interactions with the solar wind, so only little is known about the precise cosmic-ray flux in this domain. Ionization by low-energy cosmic rays is important for the chemical processes operating inside dense molecular clouds, where Lyman photons cannot penetrate [7]. Ultimately, cosmic rays are therefore likely responsible for the emergence of life. At the highest energies $(>10$ $\mathrm{EeV}$ per nuclei), the gyroradius of cosmic rays in the typical Galactic magnetic field of $3 \mu \mathrm{G}$ exceeds the thickness of the Galactic plane, so the particles are difficult to retain within the Milky Way. Particles at the very highest energies are thus generally believed to originate from extragalactic sources, yet the nature of these sources and the physical mechanism responsible for particle acceleration is still subject to much debate [11].

The origin of the Galactic component is also a matter of very active research. Following an energetic argument formulated by [8], supernova explosions could provide the necessary energy to maintain a steady state cosmic-ray flux in our Galaxy. The shock waves created by supernova explosions in the ISM provide a prolific environment for particle acceleration [9] Observation of nonthermal hard X-ray emission from supernova remnants (SNRs) demonstrates that electrons are accelerated in these shocks up to energies of $100 \mathrm{TeV}$ [10]. Gamma-ray observations are suggestive of hadron acceleration in a number of SNRs, although a definite proof for hadron acceleration by SNRs is still missing [11]. Alternative sources of cosmic rays comprise the shocks generated by the stellar winds of massive stars, pulsars and their wind nebulae $[12,13]$.

Cosmic rays in our Galaxy are best traced by high-energy gamma-ray emission $(0.1-100 \mathrm{GeV})$. The interactions of cosmic-ray particles with the interstellar medium leads to broad-band gamma-ray emission that can be traced throughout the entire Galaxy. Three physical processes contribute significantly to the diffuse high-energy gamma-ray emission from our Galaxy.

Firstly there is neutral pion decay, which dominates the gamma-ray luminosity of the Milky Way at $\mathrm{GeV}$ energies. Neutral pions $\left(\pi^{0}\right)$ are a product of hadronic interactions in the interstellar medium, mainly cosmic-ray protons colliding with the nuclei of hydrogen atoms of the interstellar gas, although also heavier nuclei contribute. After a mean lifetime of $8.4 \times 10^{-17} \mathrm{~s}, \pi^{0}$ decays into 2 gamma-ray photons with energies of $67.5 \mathrm{MeV}$ in the rest frame of the pion. This energy will be substantially Doppler shifted with respect to the Earth rest frame due to the large kinetic energies of the $\pi^{0}$ mesons in the cosmic rays. For energies that are large compared to the $\pi^{0}$ rest mass of $135 \mathrm{MeV}$, the gamma-ray spectrum mimics the cosmic-ray spectrum, and thus provides a directly measure of the cosmic-ray particle spectrum. 


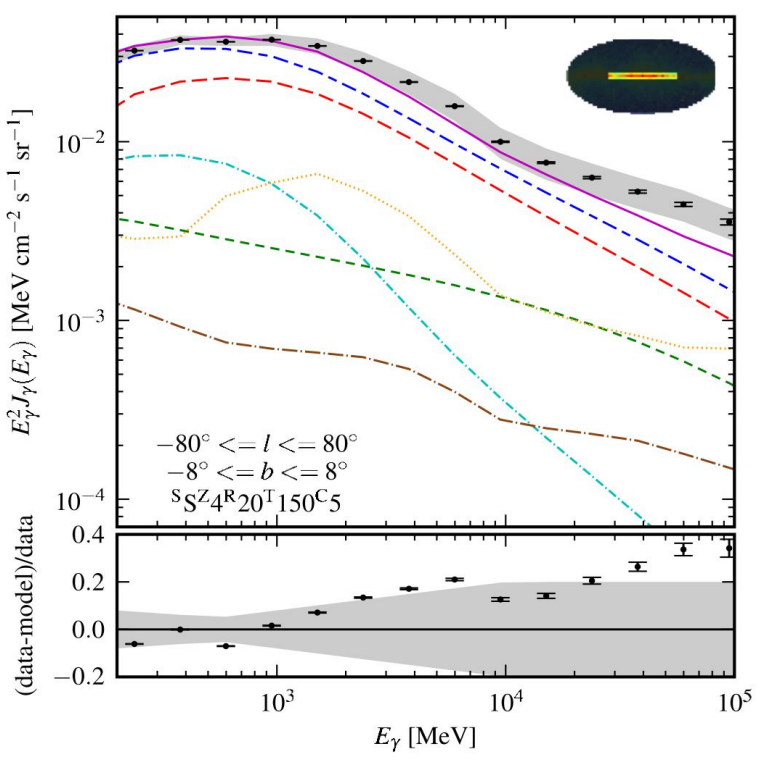

Fig. 1 Fermi-LAT spectrum of the diffuse gammaray emission from the inner Galaxy (black points) compared to a spectral model [17]. The model has been split into three basic emission components: $\pi^{0}$ decay (red, long-dashed), inverse Compton emission (green, dashed), and relativistic Bremsstrahlung (cyan, dashdotted). Also shown is the total diffuse galactic emission (blue, long-dash-dashed) and total emission (magenta, solid) including sources (orange, dotted) and isotropic background (brown, longdash-dotted). The gray region represents the systematic error in the Fermi-LAT effective area. The bottom panel shows the fractional residual (data-model)/data.

Secondly there is inverse Compton emission that arises from the interaction of cosmic-ray electrons with the interstellar radiation field and the cosmic microwave background radiation. The main contributions to the interstellar radiation field come from stars (emitting from near-infrared to ultraviolet wavelengths) and dust (emitting in the mid- to far-infrared domain) [14]. The index $\alpha$ of the resulting gammaray spectrum relates to the index $\delta$ of the electron spectrum through $\alpha=(\delta+1) / 2$, with a cutoff due to the Klein-Nishina effect that typical arises at energies of $\sim 50$ $\mathrm{TeV}$. Thus, for proton and electron distributions with comparable spectral indices, the inverse Compton emission is harder than the $\pi^{0}$ decay emission, and hence it should dominate the Galactic diffuse emission at $\mathrm{TeV}$ energies.

Thirdly there is non-thermal (or relativistic) Bremsstrahlung that arises when cosmic-ray electrons interact with the electrostatic field of atomic nuclei. The gamma-ray spectrum mimics the cosmic-ray spectrum, and thus it provides a directly measure of the cosmic-ray particle spectrum. The index $\alpha$ of the resulting gamma-ray spectrum mimics again that of the particles (i.e. $\alpha=\delta$ ). While at $\mathrm{GeV}$ energies relativistic Bremsstrahlung is believed to be negligible, it may become dominant at energies below the $\pi^{0}$ creation threshold [15] (but see [16] who argue that relativistic Bremsstrahlung is negligible in the $\mathrm{MeV}$ energy range).

Figure 1 illustrates the contribution of the three components to the Galactic diffuse emission as seen from the inner Galaxy by the Large Area Telescope (LAT) aboard the Fermi satellite after 21 months of observations [17]. In addition to the diffuse emission components, the figure also shows the point source (orange, dot- 


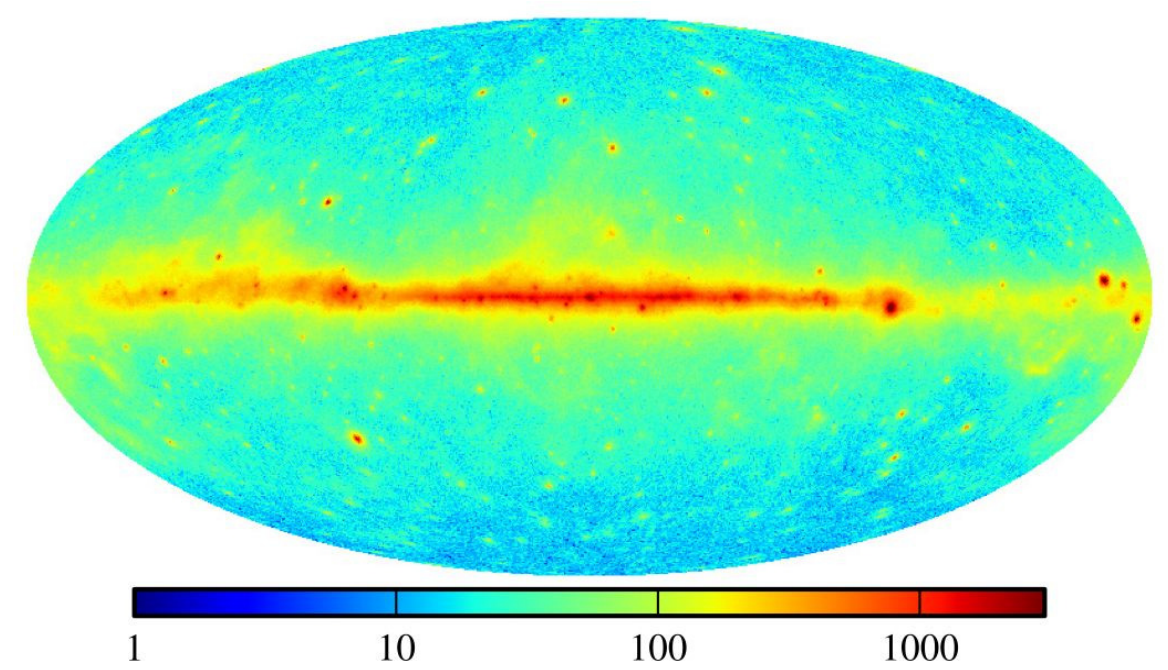

Fig. 2 High-energy gamma-ray $(0.2-100 \mathrm{GeV})$ allsky map obtained by Fermi-LAT after 21 months of observations [17].

ted) and isotropic background (brown, long-dash-dotted) contributions, which are negligible. Neutral pion decay (red, long-dashed) dominates the Galactic diffuse emission, followed by relativistic Bremsstrahlung (cyan, dash-dotted) at energies $\lesssim 2 \mathrm{GeV}$ and inverse Compton emission (green, dashed) at higher energies. Within the systematic uncertainties of the analysis (grey area), the model explains the data reasonably well, although some significant deviations occur above a few tens of $\mathrm{GeV}$.

Figure 2 shows the $0.2-100 \mathrm{GeV}$ gamma-ray allsky map as seen by Fermi-LAT over the same 21 months of observations [17]. About $~ 80 \%$ of the photons in this map are attributed to cosmic-ray interactions in our Galaxy, visible as a bright and diffuse emission structure running along the Galactic plane. The remaining photons arise from individual sources, situated either in or outside our Galaxy. Among the Galactic sources we find pulsars and their wind nebulae, supernova remnants, globular clusters, binary systems composed of a compact object and a high-mass stellar component, and one nova [85]. The extragalactic sources are all galaxies. Most of them are powered by a supermassive black hole in their centre and show radio jets; they manifest as blazars or radio galaxies [19]. A handful of the galaxies are powered by cosmic rays. These are either normal galaxies in the Local Group of galaxies, or nearby starburst galaxies [20].

The gamma-ray emissions from these normal and starburst galaxies probe cosmicray properties and cosmic-ray physics in different systems and environments. Nearby systems can eventually be resolved, allowing to infer the cosmic-ray density distribution in the galaxy. We will demonstrate this with the recent Fermi-LAT observations of the Large Magellanic Cloud [21]. Even if not resolved, the integrated gamma-ray luminosity of the galaxy bears some information about the average 
cosmic-ray density, allowing to compare cosmic-ray injection rates to galaxy properties. We will demonstrate this by comparing the gamma-ray properties of the normal and starburst galaxies detected by Fermi-LAT [20, 22]. And measuring the gamma-ray spectra provides information about the cosmic-ray particle spectra and the emission components in the galaxies, allowing for inferences about cosmic-ray physics and diffusion. This will be illustrated by discussing the gamma-ray spectra of the normal and starburst galaxies detected in high-energy or very high-energy gamma-ray so far [20].

\section{Some historical remarks}

High-energy gamma-ray emission from the plane of our Galaxy has been predicted in the late fourties and early fifties [23, 24, 25, 26], and a first gamma-ray sky map (made out of 22 events!) has been derived from data of the Explorer XI satellite, indicating a putative "tendency for the events to cluster about the galactic plane" [27]. A clear detection of the Galactic plane emission has been achieved with the OSO3 satellite in the late sixties, measuring an intensity above $100 \mathrm{MeV}$ of $5 \times 10^{-4}$ $\mathrm{ph} \mathrm{cm} \mathrm{cm}^{-2} \mathrm{~s}^{-1} \mathrm{rad}^{-1}$ towards the inner Galaxy. This intensity was in excess of expectations based on the cosmic-ray density observed at Earth, indicating a "remarkably great increase" of the cosmic-ray density towards the inner regions of the Galaxy [28]. Based on the 621 sky events measured by OSO-3, a first sky map that clearly evidenced the Galactic plane was presented by [29].

Following these early results, the SAS- 2 and COS-B satellites considerably deepened our view of the Galaxy in high-energy gamma-rays. Variations of the cosmicray density throughout the Galactic disk were needed to explain the observational data [30], and overall, gamma-ray intensity variations along the Galactic plane appeared now clearly related to the spiral arm structure of the Milky Way [31]. The evidence was growing that cosmic-ray nuclei of a few $\mathrm{GeV}$ were likely of Galactic origin [32]. Gamma-rays were established as a tracer of the total gas column density in the local ISM [33], and were even used to map the still poorly known molecular hydrogen distribution of the Galaxy [34]. Once the molecular hydrogen had been mapped accurately using large-scale CO surveys [35], the gamma-ray observations were used to calibrate the CO-to- $\mathrm{H}_{2}$ conversion factor [36]. Using the kinematic information in the radio surveys of neutral and molecular hydrogen, [37] derived for the first time the radial distribution of high-energy gamma rays in the outer Galaxy, suggesting that the density of $\mathrm{GeV}$ cosmic-ray nuclei remains surprisingly constant beyond the solar circle. The studies have subsequently been extended to the entire Galaxy $[38,39]$, confirming the weakness of the radial cosmic-ray density gradient. From the distribution of potential cosmic-ray sources (e.g., SNRs or pulsars) in the Galaxy a much steeper radial gradient had been predicted, and until now, the weakness of the radial cosmic-ray density gradient, known as the "cosmic-ray gradient problem", is not satisfactorily explained (see below). 
With the launch of the EGRET telescope aboard the Compton Gamma-Ray Observatory (CGRO) in 1991, precision measurements of Galactic diffuse gamma-ray emission became possible. EGRET enabled for the first time studies of individual molecular clouds which showed that the $\mathrm{CO}-$ to- $\mathrm{H}_{2}$ conversion factors and gammaray emissivities are comparable between the clouds and the intercloud medium $[40,41,42]$. The analysis of data from the entire Galaxy indicated that no significant spectral variations exist below $4 \mathrm{GeV}$, suggesting that the cosmic-ray electron to proton ratio does not vary significantly throughout the Galaxy [43]. At higher energies, the data indicate some spectral variations which may arise from cosmicray diffusion and/or escape processes. Most features of the observed gamma-ray emission have been reproduced using a model that assumed coupling between the matter and cosmic-ray densities, based on arguments of dynamic balance between matter, magnetic fields and cosmic rays [44]. Only above $1 \mathrm{GeV}$, the observed flux exceeded the model prediction by a significant amount, a feature named the "GeV excess" [43]. An alternative modeling approach, called GALPROP, has been developed by $[14,45]$. This model emphasizes cosmic-ray propagation calculations and a larger inverse Compton contribution to the gamma radiation, and using plausible assumptions about enhanced cosmic-ray densities with respect to the local measurements, it was able to explain the $\mathrm{GeV}$ excess. More recently, [46] used EGRET data to evidence the need for an additional gas component that is unseen in radio observations to explain the morphology of the GeV gamma-ray emission. This unseen gas is referred to as the "dark gas" component.

Besides our own Galaxy, EGRET detected also for the first time an external normal galaxy in GeV gamma-rays: the Large Magellanic Could (LMC) [47]. The signal from this Galaxy had been searched for since the early days of gamma-ray astronomy [27], but only EGRET had sufficient sensitivity to capture the faint emission. The few photons detected from the LMC combined with the limited angular resolution of EGRET did not allow for a spatial mapping of the emission, yet it was inferred that the emission morphology is consistent with that of the radio emission. The integrated gamma-ray flux seen from the LMC was consistent with predictions based on a model using the principles of dynamic balance and containment [48], and from this agreement, [47] concluded that the level of cosmic rays in the LMC is comparable to that in our Galaxy.

In contrast, the Small Magellanic Cloud (SMC) was not detected by EGRET. The upper-limit thus derived allowed to clearly dismiss a metagalactic origin for the bulk of cosmic rays with energies $10^{15}-10^{16} \mathrm{eV}$ [49], settling a long standing question in cosmic-ray physics.

No other normal galaxy was seen by EGRET, and also the most actively starforming galaxies, such as starbursts, luminous or ultraluminous infrared galaxies (LIRGs or ULIRGs), escaped detection. Upper limits on the flux from the Andromeda galaxy (M 31) have been published by [50], upper limits for other galaxies can be derived from Fig. 3 of [51]. Also the stacking analysis of EGRET observations at the positions of LIRGs and ULIRGs did not reveal any evidence for gammaray emission from this class of objects [52]. 


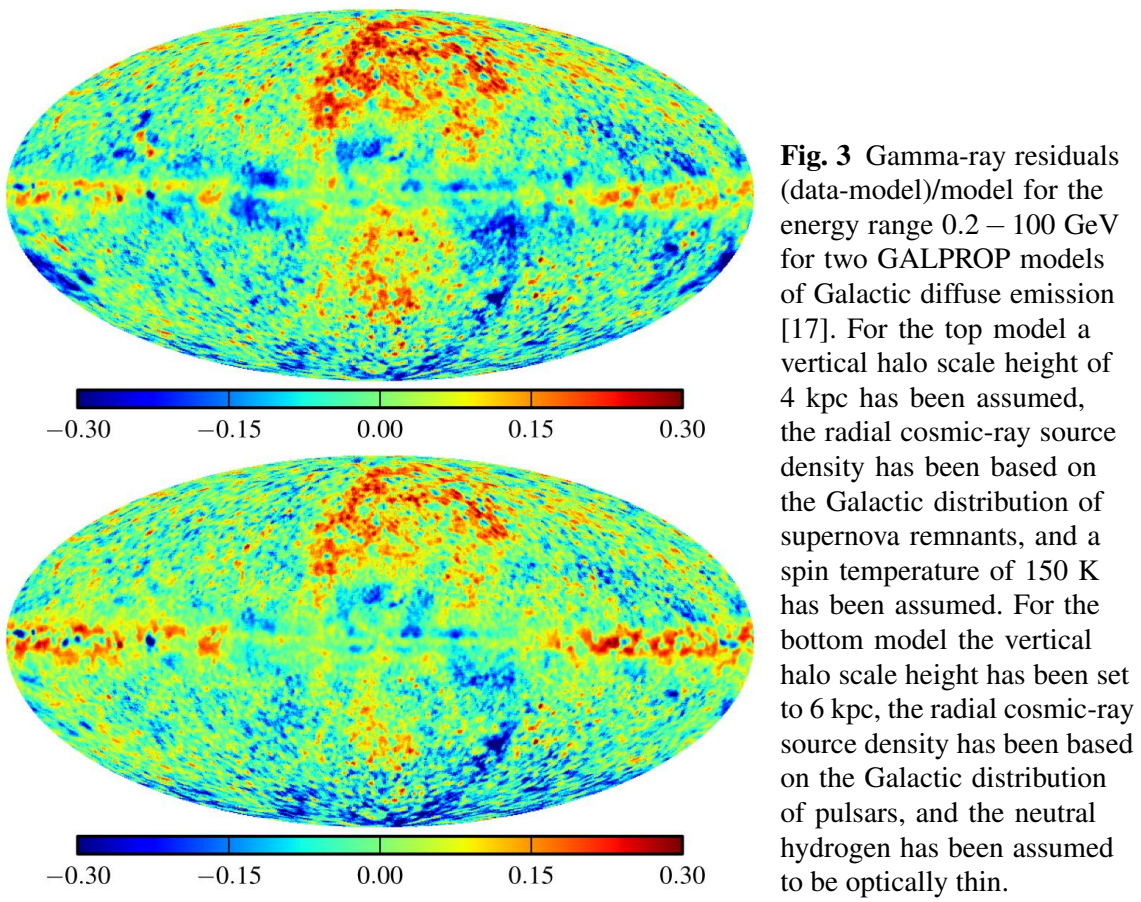

\section{The Milky Way}

Fermi-LAT provides a view of the entire gamma-ray sky from $30 \mathrm{MeV}$ to beyond $100 \mathrm{GeV}$ with a sensitivity surpassing that of EGRET by more than an order of magnitude [53]. It is thus an excellent instrument to study the diffuse gamma-ray emission from our Galaxy.

One of the first observations of the telescope was the non-confirmation of the $\mathrm{GeV}$ excess observed by EGRET. The Fermi-LAT spectrum that is observed at intermediate Galactic latitudes is in fact consistent with diffuse emission models based on the measured cosmic-ray spectra [54]. The same is true for the spectrum observed from the inner Galaxy (see Fig. 1). The GeV excess was thus explained as a systematic error in the calibration of the EGRET telescope.

The emissivity of nearby atomic hydrogen was also found to agree with expectations assuming measured cosmic-ray spectra [55], confirming earlier analyses by SAS-2, COS-B and EGRET that, however, were hampered by the uncertain subtraction of point-source contributions.

Analysis of data for the second [56] and third Galactic quadrants [57] confirmed the cosmic-ray gradient problem, showing that the decrease of the gamma-ray emissivity towards the outer Galaxy is smaller than expected from cosmic-ray diffusion models. The problem is particularly visible in all sky residual maps that are obtained after subtracting plausible cosmic-ray diffusion models that had been fitted to the 
Fermi-LAT data from the observed diffuse Galactic emission (see Fig 3). Towards the outer regions of the Galaxy (i.e. towards the left and right boundaries of the maps), significant positive emission residuals remain along the Galactic plane, irrespectively of the model assumptions. The fractional residuals are generally smaller towards the inner region of the Galaxy, which is no surprise because this area shows the strongest signal on the sky, and hence is driving the parameter fit. The main message that can be retained from Fig. 3 is that we still do not understand the large scale distribution of the gamma-ray emissivity in the Galaxy, which can be due to our limited understanding of cosmic-ray physics (source distribution, diffusion properties, size of cosmic-ray halo), or due to our incomplete knowledge of the interstellar gas distributions and densities, or due to both.

In addition to the enhanced emission towards the outer Galaxy, the all sky residual maps show notable excess emission above and below the Galactic centre direction. Towards the north, the residual emission is dominated by emission correlated with Loop I, a nearby giant radio loop spanning over 100 degrees and centered on the Sco-Cen OB association [58]. The Loop I emission may be the inverse Compton counterpart of the synchrotron emission seen in the Haslam $408 \mathrm{MHz}$ radio map [59], with possible contributions from $\pi^{0}$ decay gamma rays associated with neutral hydrogen [61].

Superimposed on the Loop I emission, and also visible south of the Galactic plane, is an emission excess with a bipolar structure that obeys a harder gamma-ray spectrum than the diffuse emission from the Galactic plane [60]. This bipolar structure has been dubbed the "Fermi bubbles" and presents $\sim 5 \%$ of the total Galactic gamma-ray luminosity between 0.1 and $100 \mathrm{GeV}$ [61]. ${ }^{1}$ The gamma-ray emission in this structure seems most likely to originate from inverse Compton scattering, since the required electron cosmic-ray population can also naturally generate the WMAP haze as a synchrotron signal. ROSAT X-ray measurements suggest that the bubbles are hot and hence underdense regions, and thus argue against the gamma rays originating from bremsstrahlung or $\pi^{0}$ decay [61]. The origin of the bipolar structure is much less clear. Scenarios tempting to explain the creation of the bipolar structure invoke a recent activity period of the supermassive black hole at the Galactic centre [61, 63, 64], a recent starburst towards the Galactic centre [61, 65], or more exotic phenomena like annihilating dark matter [66,67]. In this context it should be mentioned that the possible detection of a $130 \mathrm{GeV}$ gamma-ray line in Fermi-LAT data of the Galactic centre [69] considerably stimulates the ideas about dark matter annihilating in the central regions of the Milky Way. On the other hand, [68] recently identify a gamma-ray cocoon feature within the southern bubble, a jetlike feature along the cocoon's axis of symmetry, and another directly opposite the Galactic centre in the north. If the detection of such a jet-like feature is confirmed, the hypothesis that the Fermi bubbles are the result of recent AGN-like activity at the centre of our Galaxy would be considerably strengthened.

\footnotetext{
${ }^{1}$ The discovery paper [60] called this emission the "Fermi haze", in analogy to the "WMAP haze" that has been discovered by the same group in the data of the Wilkinson Microwave Anisotropy Probe (WMAP) [62].
} 

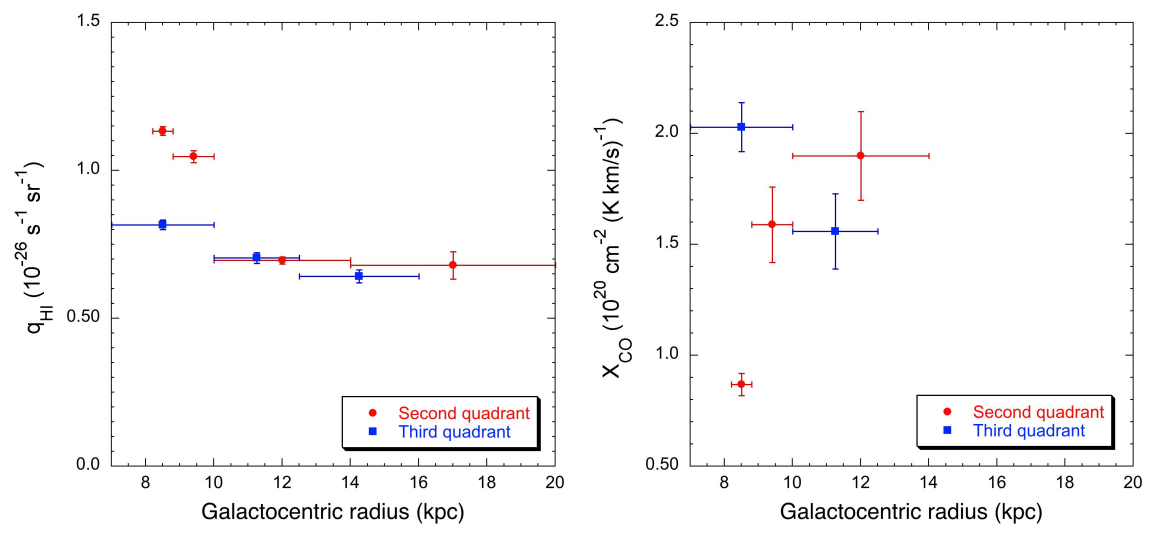

Fig. 4 Gamma-ray emissivities $>200 \mathrm{MeV}$ (left panel) and CO-to- $\mathrm{H}_{2}$ conversion factors (right panel) as function of Galactocentric radius as determined using Fermi-LAT data for the second and third Galactic quadrant [56, 57]. A spin temperature of $125 \mathrm{~K}$ has been assumed to derive the values.

But let's come back to the cosmic-ray gradient problem. The left panel of Fig. 4 shows the $>200 \mathrm{MeV}$ gamma-ray emissivities as function of Galactocentric radius that have been derived for the second and third Galactic quadrant using Fermi-LAT data $[56,57]$. In the right panel we show the CO-to- $\mathrm{H}_{2}$ conversion factors $X_{\mathrm{CO}}$ that have been computed from the $\mathrm{CO}$ to $\mathrm{H}_{\mathrm{I}}$ emissivity ratio using $X_{\mathrm{CO}}=q_{\mathrm{CO}} /\left(2 q_{\mathrm{HI}}\right)$. The left panel illustrates the rather shallow decline of gamma-ray emissivities beyond $10 \mathrm{kpc}$ which suggests a small cosmic-ray gradient in the outer Galaxy. In contrast, $\mathrm{H}$ I emissivity values in the solar vicinity show quite some dispersion. One may be tempted to conclude that the dispersion suggests variations in the local cosmicray density, but a comparison with the right panel indicates an alternative explanation. Regions with high $\mathrm{H}_{\mathrm{I}}$ emissivity show a surprisingly low $X_{\mathrm{CO}}$ ratio, which may indicate that $q_{\mathrm{HI}}$ has been overestimated by the analysis. In the general analysis procedure, which goes back to the COS-B era [37], a linear combination of gas map templates is fitted to the data. The parameters that are adjusted are the normalization factors $q_{\mathrm{HI}}$ and $q_{\mathrm{CO}}$, representing the emissivity of neutral and molecular hydrogen, respectively (in practice, additional components are added to represent dark gas, the isotropic background, and point sources in the field [56]). As the fit matches the templates to the data, an increase of $q_{\mathrm{HI}}$ leads necessarily to a reduction of $q_{\mathrm{CO}}$, making $X_{\mathrm{CO}}$ very sensitive to the precise balance between $q_{\mathrm{HI}}$ and $q_{\mathrm{CO}}$. Both parameters are well defined if the morphology of the gas templates is very distinctive, but increasing crosstalk is seen for $\mathrm{H}$ I and $\mathrm{CO}$ maps that show strong resemblance (see for example Fig. 3 in [42]). The impact of the spin temperature on derived $\mathrm{H}_{\mathrm{I}}$ column densities, and the possible presence of dark gas considerably complicates the situation [70]. It remains to be seen whether these effects can explain some of the features seen in Fig. 4 . In the meantime, claims of $X_{\mathrm{CO}}$ gradients and variations $[42,56,70]$ should be considered with caution. 


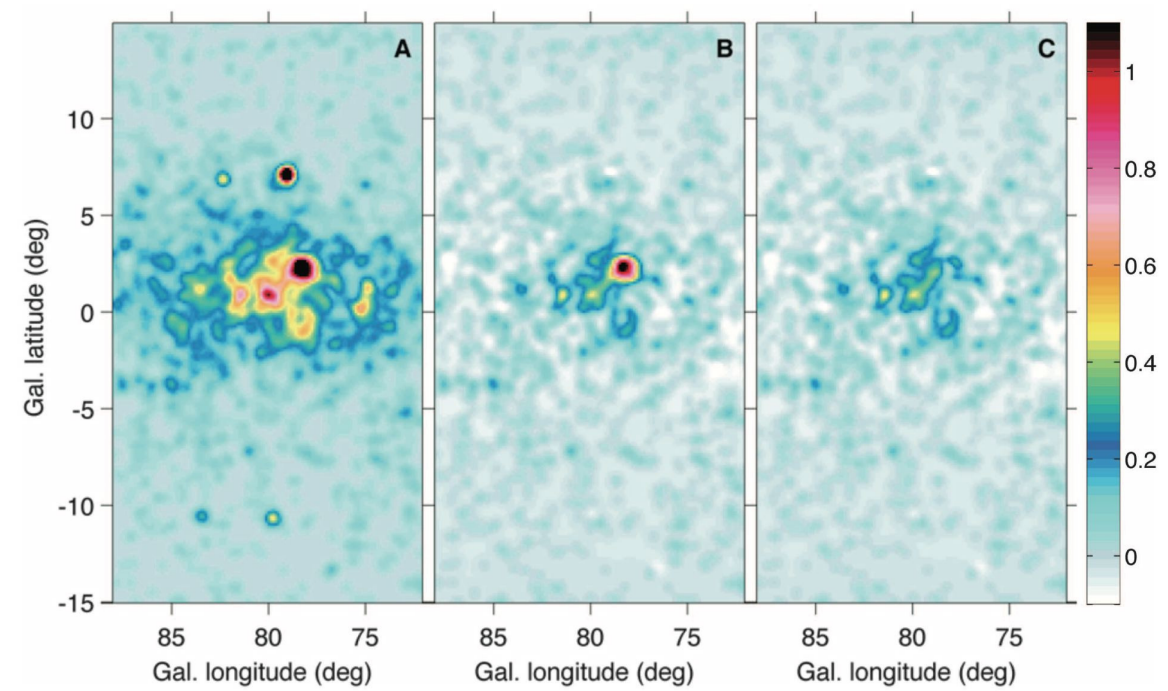

Fig. 5 Fermi-LAT counts maps in the $10-100 \mathrm{GeV}$ band smoothed with a $\sigma=0.25^{\circ}$ Gaussian kernel [72]. From left to right, the panels show the total emission (A), the emission after subtraction of the interstellar background and all known sources but $\gamma$ Cygni (B), and after further removal of the extended emission from $\gamma$ Cygni (C).

Massive star forming regions have been postulated since a while as potential sites of cosmic-ray induced gamma-ray emission [73, 12], and recent observations with the Milagro Gamma Ray Observatory in the TeV domain have been interpreted as evidence for active cosmic-ray sources accelerating hadrons in the Cygnus $\mathrm{X}$ region [74]. Using two years of Fermi-LAT data, [71] have investigated the diffuse gamma-ray emission from the Cygnus region, and find a gamma-ray emissivity and $X_{\mathrm{CO}}$ factor that is in agreement with the values measured in the local interstellar medium. So despite the conspicuous star formation activity and high masses of the interstellar clouds, the cosmic-ray population in the Cygnus complex averaged over a few hundred parsecs appears similar to that of the local interstellar space.

Nevertheless, above $10 \mathrm{GeV}$, an additional hard spectral component has been evidenced in the Fermi-LAT data that spatially correlates well with the interstellar cavity that has been carved out through ionization, radiation pressure, and the winds of the massive stellar population of the Cyg OB2 cluster [72]. Figure 5 presents three maps of the Cygnus region, showing the residuals after progressively removing known emission components. The excess remaining in the right panel (C) coincides well with the Cygnus superbubble. The total $1-100 \mathrm{GeV}$ luminosity of this excess has been estimated to $(9 \pm 2) \times 10^{34} \mathrm{erg} \mathrm{s}^{-1}$ [72], which is surprisingly small compared to the mechanical wind power of $4 \times 10^{38} \mathrm{erg} \mathrm{s}^{-1}$ that is available from the Cyg OB2 association [75]. If Cyg OB2 were at the origin of the observed emission, only $\sim 0.02 \%$ of its available kinetic power would be tapped to produced the observed gamma-ray emission. 


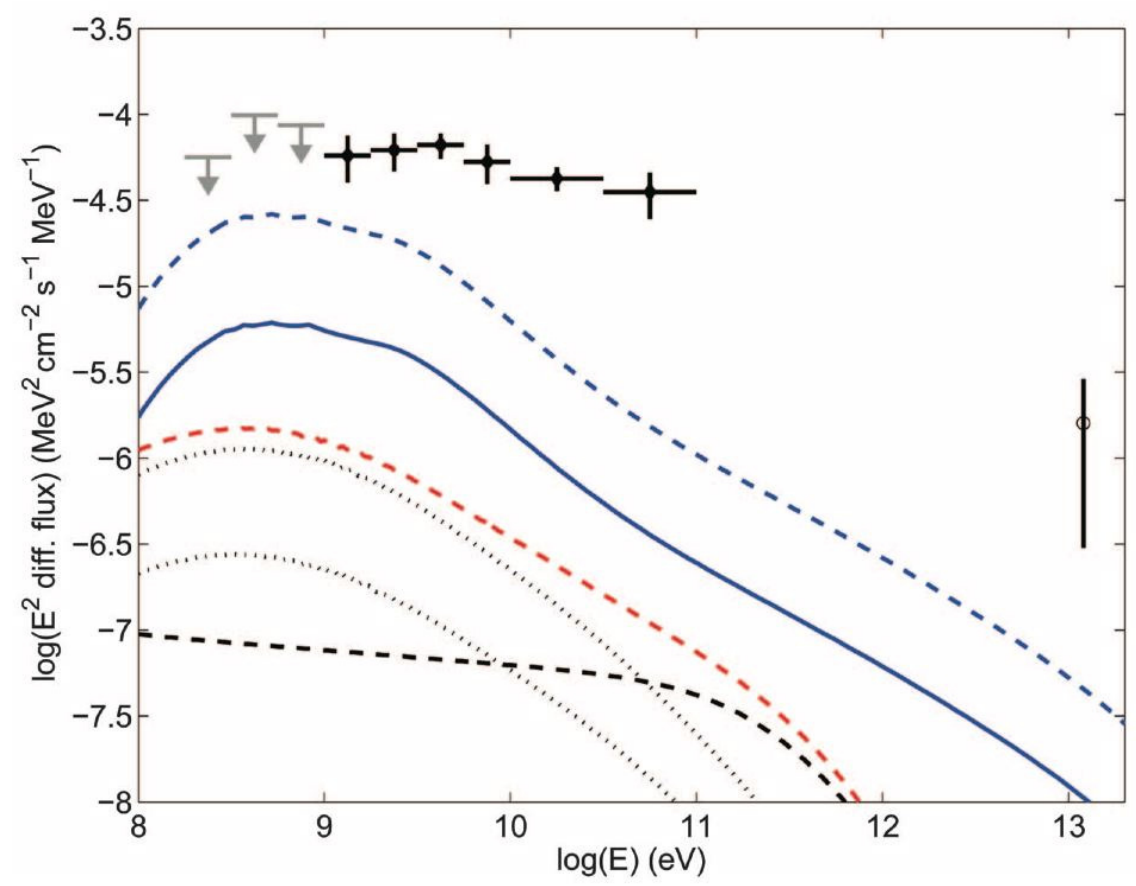

Fig. 6 Energy spectrum of the cocoon emission [72]. The $1 \sigma$ errors are statistical, $2 \sigma$ upper limits are given below $1 \mathrm{GeV}$. The open circle shows the Milagro flux, integrated over $78.7^{\circ}<l<81.7^{\circ}$ and $-0.4^{\circ}<b<2.6^{\circ}$, and corrected for the extrapolated contribution of the $\mathrm{TeV} \mathrm{J} 2032+4130$ source at energies $>10 \mathrm{TeV}$. The blue curves show the expected $\pi^{0}$ decay emission based on the locally measured cosmic-ray spectrum pervading the ionized gas for electron densities $10 \mathrm{~cm}^{-3}$ (solid) and $2 \mathrm{~cm}^{-3}$ (dashed). The black curves show the expected inverse Compton emission based on the locally measured cosmic-ray spectrum, upscattering the stellar light from Cyg OB2 (upper dotted curve), NGC 6910 (lower dotted curve), and the interstellar radiation present in the PDRs (dashed curve). The red curve sums all inverse Compton emissions.

The energy spectrum of the excess emission is presented in Fig. 6, demonstrating the hardness of the spectrum when compared to Galactic diffuse emission components. The expected contributions of $\pi^{0}$ decay and inverse Compton emission based on the locally measured cosmic-ray spectrum are superimposed as lines, illustrating that the observed emission is in excess of and harder than model predictions using plausible assumptions on gas densities and interstellar radiation fields in the region. The excess argues for an additional cosmic-ray component to be present in Cygnus, the hardness points to freshly accelerated particles [72]. Interestingly, although separated by a large gap without any data, the flux point obtained by Milagro seems to fit the extrapolation of the Fermi-LAT spectrum to TeV energies.

It remains to be seen whether the cocoon of freshly accelerated cosmic rays detected by Fermi-LAT in the Cygnus superbubble can indeed be explained by cosmicray acceleration in the stellar winds of the massive OB stars of young stellar clusters. 
More such cocoons should be found within our Galaxy to confirm this scenario, yet Cyg OB2 is one of the most massive young stellar clusters known in our Galaxy, and definitely the closest one of such mass, hence other potential candidates may be too faint to be detectable by current instruments. Fermi-LAT has however detected gamma rays from another massive star cluster situated in the nearby LMC galaxy [21], and we now turn to these observations to see how they fit in our global understanding of cosmic-ray acceleration and propagation in the Milky Way.

\section{The Large Magellanic Cloud}

As targets for studies of cosmic-ray physics, nearby galaxies have the advantage of being viewed from outside, and so line of sight confusion, which complicates studies of emission from the Galactic disk, is diminished. This advantage is, however, somewhat offset by the limitations of angular resolution and sensitivity of the instrument. The LMC is thus an excellent target for studying the link between cosmic-ray acceleration and gamma-ray emission since the galaxy is nearby $(D \approx 50 \mathrm{kpc} ;[76,77])$ and thus reasonably bright, has a large angular extent of $\sim 8^{\circ}$ and thus is easily resolvable, and is seen at a small inclination angle of $i \approx 20^{\circ}-35^{\circ}[78,79]$, which avoids source confusion. In addition, the LMC is relatively active, housing many supernova remnants, bubbles and superbubbles, and massive star-forming regions that are all potential sites of cosmic-ray acceleration.

Fermi-LAT has provided the first spatially resolved gamma-ray image of the LMC [21], that is presented in Fig. 7. A striking feature of this image is that the gamma-ray emission does not correlate well with the gas distribution of the galaxy. Under the conventional assumption that a galaxy is filled up with a "sea" of cosmicray particles that is homogenized through the effects of convection and diffusion in galactic magnetic fields [80], one would predict that the gamma-ray image of the LMC should in first order be a proxy of the gas distribution in the galaxy, modulated eventually by a large scale cosmic-ray density gradient. In contrast, a substantial fraction of the LMC surface area appears devoid of gamma rays, and the emission shows considerable structure, with a single bright feature dominating the image. Interestingly, this features coincides with 30 Doradus, one of the most active starburst regions in the entire Local Group of galaxies [81].

A comparison of the observed distribution of gamma rays to tracers of interstellar emission components has revealed that the emission morphology correlates well with the ionized gas component, while neutral atomic or molecular hydrogen show considerably different morphologies [21]. This result even holds if the 30 Doradus region is excluded from the correlation analysis. This finding does not imply, however, that the gas with which the cosmic rays interact is necessarily ionized, it simply shows that the spatial distribution of the LMC gamma-ray emissivity resembles the spatial distribution of ionized gas. As interstellar ionization is primarily provided by UV photons from $\mathrm{O}$ stars (i.e. stars with masses $>20 \mathrm{M}_{\odot}$ ), the distribution of ion- 


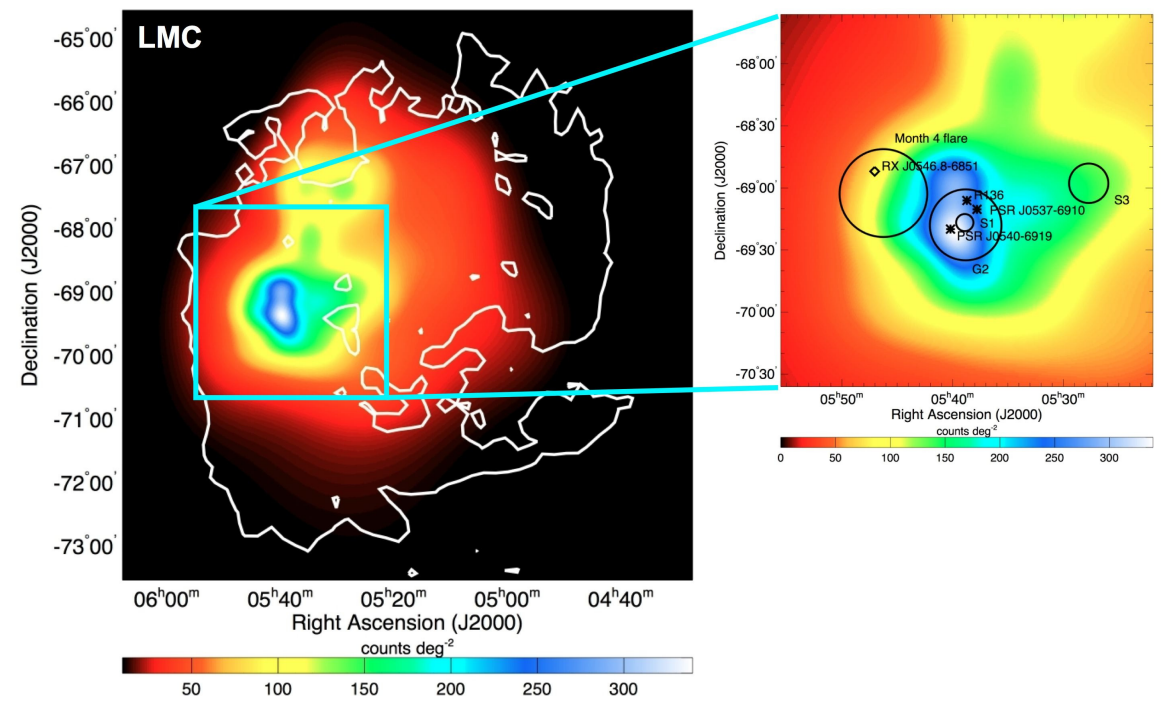

Fig. 7 Fermi-LAT image of the Large Magellanic Cloud. The image is based on a background subtracted counts map that has been adaptively smoothed to remove image noise. In the left panel, the colors show the gamma-ray emission while the white contour shows the outline of the LMC as traced by neutral hydrogen. The right panel shows a zoom into the 30 Doradus region. The circles indicate the locations of source components that have been fitted to the data. The stars mark the positions of potential gamma-ray emitting sources. The location of an observed flare in the area is also indicated. The figure has been adapted from [21].

ized gas traces the distribution of $\mathrm{O}$ stars. Consequently, the gamma-ray emissivity of the LMC correlates with the distribution of O stars.

The shortcut of attributing this correlation to cosmic-ray production by $\mathrm{O}$ stars is appealing, but in view of the results obtained for our own Galaxy, and in particular those of the Cygnus region [71, 72], some caution is appropriate. Cyg OB2 houses about $120 \mathrm{O}$ stars [82], which is, within a factor of a few comparable to the number of $\mathrm{O}$ stars found in 30 Doradus [83]. The kinematic energy input of the stars in 30 Doradus should therefore also be comparable to the input provided by the Cyg OB2 stars, say about $\sim 10^{39} \mathrm{erg} \mathrm{s}^{-1}$, which is in line with studies of the kinematic structure of the region [84]. The observed $>100 \mathrm{MeV}$ gamma-ray flux from 30 Doradus amounts to $(4.6 \pm 0.5) \times 10^{-11} \mathrm{erg} \mathrm{s}^{-1} \mathrm{~cm}^{-2} \mathrm{sr}^{-1}$, corresponding to a luminosity of $(1.4 \pm 0.1) \times 10^{37} \mathrm{erg} \mathrm{s}^{-1}$. Thus, about $\sim 1 \%$ of the mechanical luminosity of 30 Doradus are required to explain the observed gamma-ray emission. Although not excessive, this fraction is 50 times larger than the fraction inferred from the cocoon emission in Cygnus [72]. So if the 30 Doradus gamma rays are attributed to cosmic rays, there must be something fundamentally different in 30 Doradus compared to the most massive star-forming regions in the Milky Way.

But maybe there exists a simpler explanation. Massive star forming regions are rich in remnants of stellar explosions, such as supernova shells or pulsars. In particular, pulsars are known to be bright gamma-ray emitters that form the dominant 
$\mathrm{GeV}$ source population of normal galaxies [85], and numerous pulsars are found in the Galactic star forming regions Cygnus and Carina. Pulsars are also known in 30 Doradus, two of them being among the three most powerful pulsars known (PSR J0537-6910 and PSR J0540-6919). Although no gamma-ray pulsations have so far been detected from these objects [21], it would be surprising if pulsars would not contribute to the gamma-ray emission seen from 30 Doradus. The question is: can pulsars explain most of the gamma-ray flux seen from 30 Doradus?

The Crab is the most powerful gamma-ray pulsar known in the Milky-Way, yet with a $>100 \mathrm{MeV}$ gamma-ray luminosity of $\sim 6 \times 10^{35} \mathrm{erg} \mathrm{s}^{-1}$ [86], it could only account for $\sim 1 / 20$ of the observed luminosity from 30 Doradus. Thus, at the distance of the LMC, the Crab would only be a faint source, barely detectable with Fermi-LAT. Explaining the 30 Doradus emission with pulsars would either require tens of Crab-type pulsars in that area, or one pulsar which is at least ten times more luminous in gamma rays than the Crab. There is indeed some headroom for the existence of more luminous gamma-ray pulsars, as the Crab only converts a tiny fraction $(\sim 0.1 \%)$ of its rotational power into gamma rays [86]. In this context we note that H.E.S.S. recently detected $\mathrm{TeV}$ gamma rays from the pulsar wind nebula of PSR J0537-6910 in 30 Doradus [87], inferring a TeV luminosity for the nebula that is $\sim 14$ times superior to that of the Crab nebula at $\mathrm{TeV}$ energies. If the nebula $\mathrm{TeV}$ luminosity is linked to the pulsar $\mathrm{GeV}$ luminosity (which is not implausible because both are powered by the spin-down of the pulsar), PSR J0537-6910 would be a prime candidate to explain the $\mathrm{GeV}$ emission observed by Fermi-LAT from 30 Doradus. Ongoing searches for pulsations from this object in Fermi-LAT data may allow to settle this question in the future.

It should also be mentioned that owing to the large extension of the LMC, blazars lying along the line of sight behind the galaxy may also contribute to the observed signal. For the sensitivity reached during the first Fermi-LAT analysis of the LMC, it has been estimated that $1-2$ background blazars may exist within the boundaries of the galaxy, and indeed, a flare of gamma-ray emission had been observed near 30 Doradus that could indicate some blazar activity towards this general direction [21]. While a flaring signal can be easily excluded from the data (as has been done by [21]), steady emission from unknown background sources can only be distinguished from the overall LMC on basis of possible spectral differences. Nevertheless, it should be mentioned that no gamma-ray emission from known blazars in the field of the LMC has been detected by [21].

Having all these caveats in mind, and excluding the 30 Doradus region from the analysis due to the uncertain origin of the emission, the remaining overall emission of the LMC can be interpreted in the context of cosmic-ray induced gamma-ray emission. In that way, [21] derived an average $>100 \mathrm{MeV}$ gamma-ray emissivity of $\sim 0.4 \times 10^{-26} \mathrm{ph} \mathrm{s}^{-1} \mathrm{sr}^{-1} \mathrm{H}$-atom ${ }^{-1}$ which is about a factor of $\sim 4$ lower than the value measured in the local interstellar medium of the Milky Way [55]. The average cosmic-ray density in the LMC amounts thus to only $\sim 25 \%$ of the cosmicray density observed near Earth. This lower density can be explained by an either lower cosmic-ray production rate in the LMC with respect to our Galaxy, or by an easier escape of the cosmic rays into the intergalactic medium. 


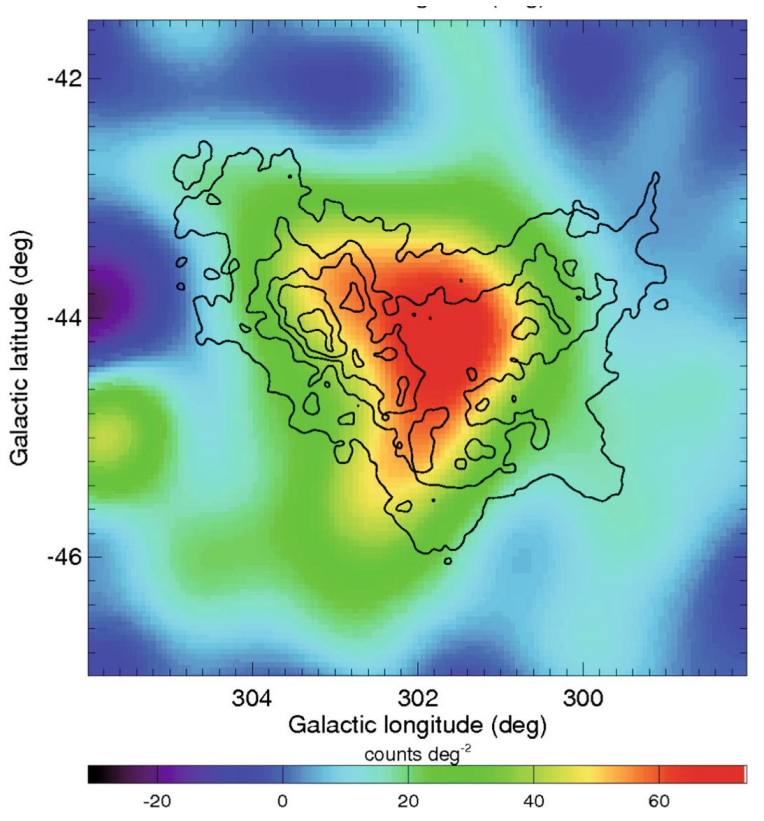

Fig. 8 Fermi-LAT image of the SMC in the $200 \mathrm{MeV}$ $20 \mathrm{GeV}$ energy band after subtraction of the celestial background [88]. The image has been smoothed by a $2 \mathrm{D}$ Gaussian kernel with $\sigma=$ $0.4^{\circ}$. The SMC is traced by $\mathrm{H}$ I column density contours.

\section{The Small Magellanic Cloud}

While before the launch of the Fermi satellite only upper limits had been derived on gamma-ray emission from the SMC [49], the LAT achieved a first detection of the galaxy [88]. Figure 8 shows the gamma-ray map of the SMC that has been obtained using 17 months of Fermi-LAT data, with contours tracing H I column density superimposed. The observed gamma-ray emission is clearly centred on the galaxy, and extends over an area of $\sim 3^{\circ}$ in diameter. Owing to the faintness of the signal combined with the small angular size of the galaxy, only limited information is so far available concerning the spatial distribution of the emission. Nevertheless, no clear correlation between the gamma-ray emission and the distribution of either neutral gas or ionized gas is observed. A possible link of the gamma-ray emission with supergiant shells has been pointed out [88].

Under the assumption that all emission can be attributed to cosmic-ray interactions with the interstellar medium, the observed integrated $>100 \mathrm{MeV}$ flux of $(3.7 \pm 0.7) \times 10^{-8} \mathrm{ph} \mathrm{cm}^{-2} \mathrm{~s}^{-1}$ implies an average cosmic-ray density in the SMC of $\sim 15 \%$ of the value measured locally in the Milky Way. Interestingly, the estimated star formation rate per unit volume in the SMC is comparable, or even larger, than that of the Milky Way, so the rate with which cosmic rays are injected in the galaxy per unit volume should be at least as large as the Galactic value. The apparently lower average cosmic-ray density in the SMC then implies that the cosmic-ray confinement time is lower or the cosmic-ray diffusion coefficient is larger in comparison to the Milky Way. Alternatively, non steady-state scenarios or the confinement 


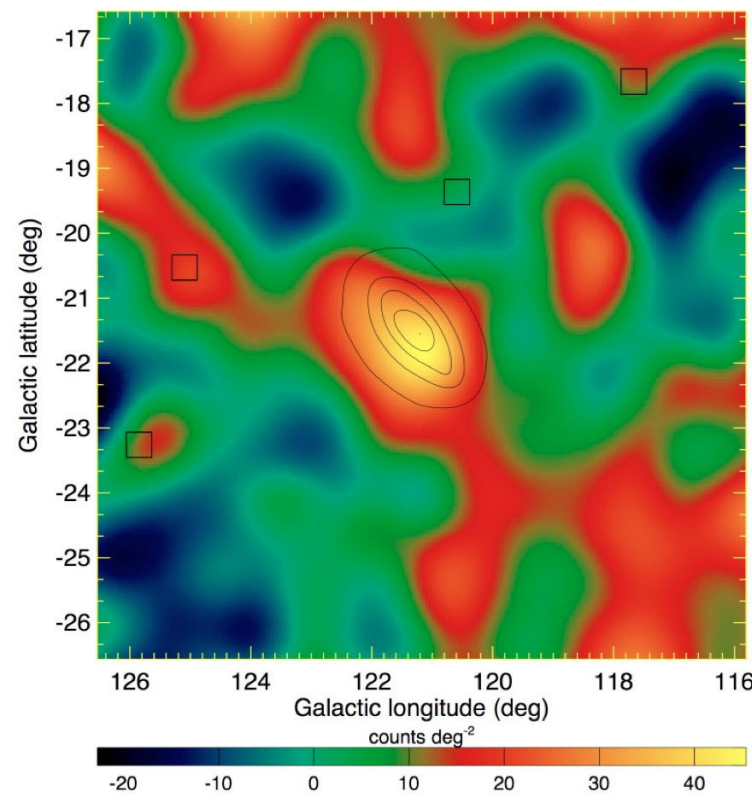

Fig. 9 Fermi-LAT image of M 31 in the $200 \mathrm{MeV}$ $20 \mathrm{GeV}$ energy band after subtraction of the celestial background and nearby point sources [22]. The image has been smoothed by a $2 \mathrm{D}$ Gaussian kernel with $\sigma=0.5^{\circ}$. M 31 is traced by contours of $100 \mu \mathrm{m}$ infrared emission that have been convolved with the LAT point spread function. The square symbols indicate the locations of the point sources that have been subtracted from the data.

of cosmic-ray in the hot and tenuous interiors of large superbubbles may also account for the observations without implying cosmic-ray densities below the Galactic one [88].

In any case, the relatively low gamma-ray flux combined with the observed star formation rate implies that the contribution of point sources to the overall gammaray emission could be significant in the SMC. By making plausible assumptions on the average luminosity and the expected number of gamma-ray pulsars that may be present in the SMC, [88] estimate that between $\sim 25 \%$ and $100 \%$ of the observed flux may be attributed to the combined emission of gamma-ray pulsars. If pulsars contribute indeed significantly to the emission, the average cosmic-ray density deduced from the observations would be even lower, reinforcing the idea that cosmic-ray escape from the SMC is amplified with respect to our Galaxy.

\section{Other Local Group galaxies}

Fermi-LAT has also provided the first detection of gamma-ray emission from the nearby Andromeda galaxy [22]. Figure 9 shows the image of the region around M 31 that has been derived using almost 2 years of continuous sky survey observations. With a significance of $5 \sigma$, the signal is at the limit of what is detectable by the LAT. Although the gamma-ray image indicates some elongation of the emission that follows the morphology of the galaxy, the current data can not allow to discriminate between point-like and extended emission. 
Assuming that the entire gamma-ray emission originates from cosmic-ray interactions with the interstellar medium of $\mathrm{M} 31$, the observations indicate an average cosmic-ray density that amounts to about half of the Galactic value [22]. As the estimated average star formation rate of M 31 is inferior to that of the Milky Way by about the same factor, the lower cosmic-ray density in M 31 is readily explained by a lower cosmic-ray injection rate in that galaxy.

Gamma-ray emission has also be searched for in the Local Group galaxies M 33 [22], M 81, M 83, IC 342, Maffei 1, Maffei 2, and M 94 [89] without any significant detection. The derived upper limits for these galaxies are fully compatible with a scenario where the gamma-ray luminosities scale with the integrated star formation rates in star-forming galaxies (see below).

\section{Starburst galaxies}

For the first time, Fermi-LAT has also detected GeV gamma-ray emission from starburst galaxies. First from the well known prototypical galaxies M 82 and NGC 253 [90], later also from NGC 1068 and NGC 4945 [91]. While for the first two objects it is widely accepted that the observed gamma-ray emission originates in cosmicray interactions with the interstellar medium of these galaxies, contributions from the active nucleus can not be excluded for NGC 1068 and NGC 4945 [91]. M 82 [92] and NGC 253 [93] have also been detected at TeV energies using ground-based Cherenkov telescopes, providing thus a wide spectral coverage of the gamma-ray emission that allow for inferences about the cosmic-ray spectrum to be made.

The gamma-ray luminosities of the starburst galaxies are clearly in excess to those of the Milky Way, M 31, the LMC and the SMC, reinforcing the idea that the cosmic-ray densities increase with increasing star formation rate of a galaxy [90]. Theoretically predicted gamma-ray spectra $[94,95,96,97,98]$ agree reasonably well with the observations, although the observed spectra are not extremely constraining, owing to the faintness of the gamma-ray flux seen at $\mathrm{GeV}$ and $\mathrm{TeV}$ energies.

While in the Milky Way, most cosmic-ray protons are believed to escape the Galaxy before being able to scatter inelastically off the ISM nuclei, generating $\pi^{0}$ meson, the dense ISM in starburst galaxies may provide a thick target to cosmicray protons that converts a substantial fraction of the cosmic-ray energy into pionic gamma rays [99]. Starburst galaxies have thus been suggested to be proton calorimeters [100]. Proton calorimetry would manifest by relatively hard spectral indices of $\Gamma \approx 2.0-2.4$ while proton escape leads to softer spectra with $\Gamma \approx 2.7$, as is observed for the Milky Way [101]. The fact that starburst galaxies are both detected at $\mathrm{GeV}$ and $\mathrm{TeV}$ energies points indeed towards relatively hard gamma-ray spectra, supporting the hypothesis that starburst galaxies are proton calorimeters. Alternatively, strong advective cosmic-ray losses, produced for example by strong galactic winds, will also produce hard gamma-ray spectra, so the question of whether starburst galaxies are indeed proton calorimeters is not yet definitely settled [101]. 
It should also be mentioned that contributions of point sources, in particular at $\mathrm{TeV}$ energies, may complicate the picture [101, 102]. The large star formation rates encountered in the cores of starburst galaxies will also result in large populations of supernova remnants and pulsar wind nebulae (PWNe). Although estimates based on the gamma-ray luminosities of Galactic PWNe imply that $\sim 10^{4}-10^{5}$ such objects would be needed to contribute significantly to the $\mathrm{GeV}-\mathrm{TeV}$ emission seen from M 82 and NGC 253, in cannot be ruled our that PWNe in starbursts are much more radiatively efficient in gamma rays that in the Milky Way, for example through stronger inverse Compton losses [101].

\section{The gamma-ray population of starforming galaxies}

Taking together the four galaxies from the Local Group (Milky Way, M 31, LMC, and SMC) and the four starburst galaxies (M 82, NGC 253, NGC 1068, and NGC 4945), a first sample of gamma-ray emitting star-forming galaxies exists that spans 5 orders of magnitude in star formation rate and gamma-ray luminosity. Figure 10 shows the $1-100 \mathrm{GeV}$ gamma-ray luminosities of this sample as function of the radio continuum (RC) luminosity at $1.4 \mathrm{GHz}$. The latter is an excellent tracer of the star formation rate (SFR), which is shown as the upper abscissa according to equation 2 of [103]. The plot shows also the 95\% confidence upper flux limits that have been obtained from the Fermi-LAT analysis for a sample of 69 dwarf, spiral, and luminous and ultraluminous infrared [20]. Obviously, there exists a good correlation between the integrated GeV gamma-ray luminosity and the integrated SFR of the galaxies, and also the upper limits are consistent with the correlation. A power law fit to the data (shown as red line) results in a slope of $\sim 1.1$, suggesting that the relation between $\mathrm{GeV}$ luminosity and SFR is slightly non-linear. Still, it is striking that the relation seems to hold from the SMC dwarf galaxy, with its low cosmic-ray density that may indicate a substantial loss of cosmic-ray particles, to NGC 1068, which is proposed to be a proton calorimeter [101].

For reference, Fig. 10 shows as red dashed line the expected gamma-ray luminosity of a galaxy in the calorimetric limit. To derive this line, it has been assumed that $10 \%$ of the kinetic energy released in a core-collapse supernova (taken to be $10^{51}$ erg) go into cosmic-ray nuclei. In this simplistic picture, none of the galaxies reaches in fact the calorimetric limit, and the slight non-linearity of the observed correlation may be interpreted as an increase of the calorimetric efficiency from $\sim 10-20 \%$ for dwarf galaxies to $\sim 30-50 \%$ for starburst galaxies [20]. In this scenario, the physical conditions for cosmic-ray escape vary surprisingly little among the galaxies, despite their large diversity in size, interstellar mass and density, and star formation rate.

Figure 11 presents a compilation of the observed $\mathrm{GeV}-\mathrm{TeV}$ gamma-ray spectra for all 8 galaxies [20]. The spectra are expressed in absolute luminosity, illustrating the wide spread in gamma-ray power among the detected objects. For the Milky Way, instead of a measured spectrum, a global model is shown that has been adjusted 


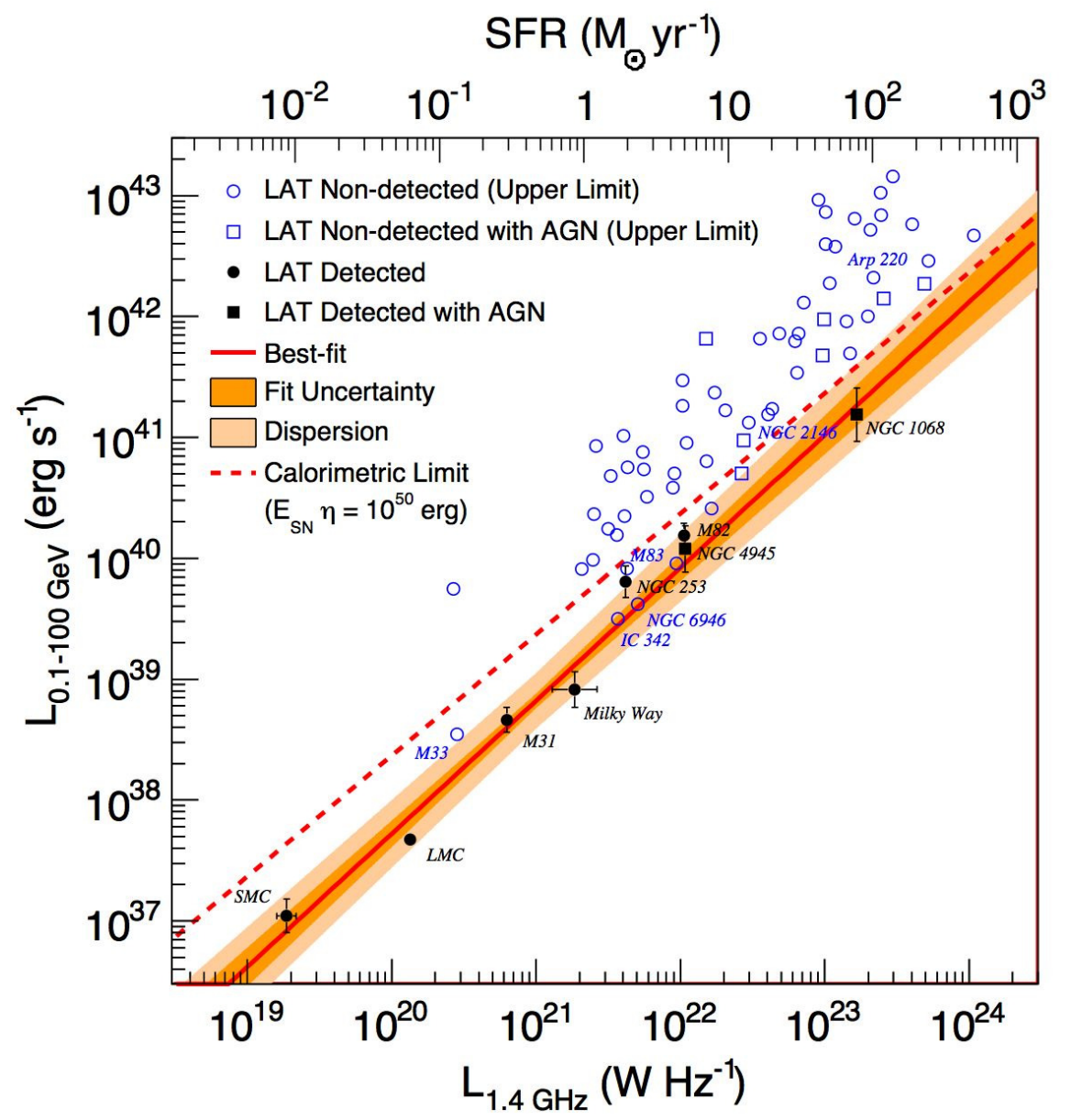

Fig. 10 Gamma-ray luminosity versus RC luminosity at $1.4 \mathrm{GHz}$. Galaxies significantly detected by the LAT are indicated with filled symbols, galaxies with gamma-ray flux upper limits are marked with open symbols, galaxies hosting AGN are shown with square markers. The upper abscissa indicates SFR estimated from the RC luminosity. The best-fit power law relation is shown by the red solid line along with the fit uncertainty (darker shaded region), and intrinsic dispersion around the fitted relation (lighter shaded region). The dashed red line represents the expected gamma-ray luminosity in the calorimetric limit assuming an average CR luminosity per supernova of $10^{50} \mathrm{erg}$ [20].

to Fermi-LAT data of diffuse Galactic gamma-ray emission (as we're observing our Galaxy from inside, it is not possible to make a model-independent estimation of the Galactic gamma-ray luminosity). The figure illustrates that the more luminous galaxies also have comparatively harder gamma-ray spectra. Whereas the powerlaw spectral indices of M 82 and NGC 253 are $2.2-2.3$ extending to TeV energies, the spectra of the LMC and SMC steepen above $\sim 2 \mathrm{GeV}$. 


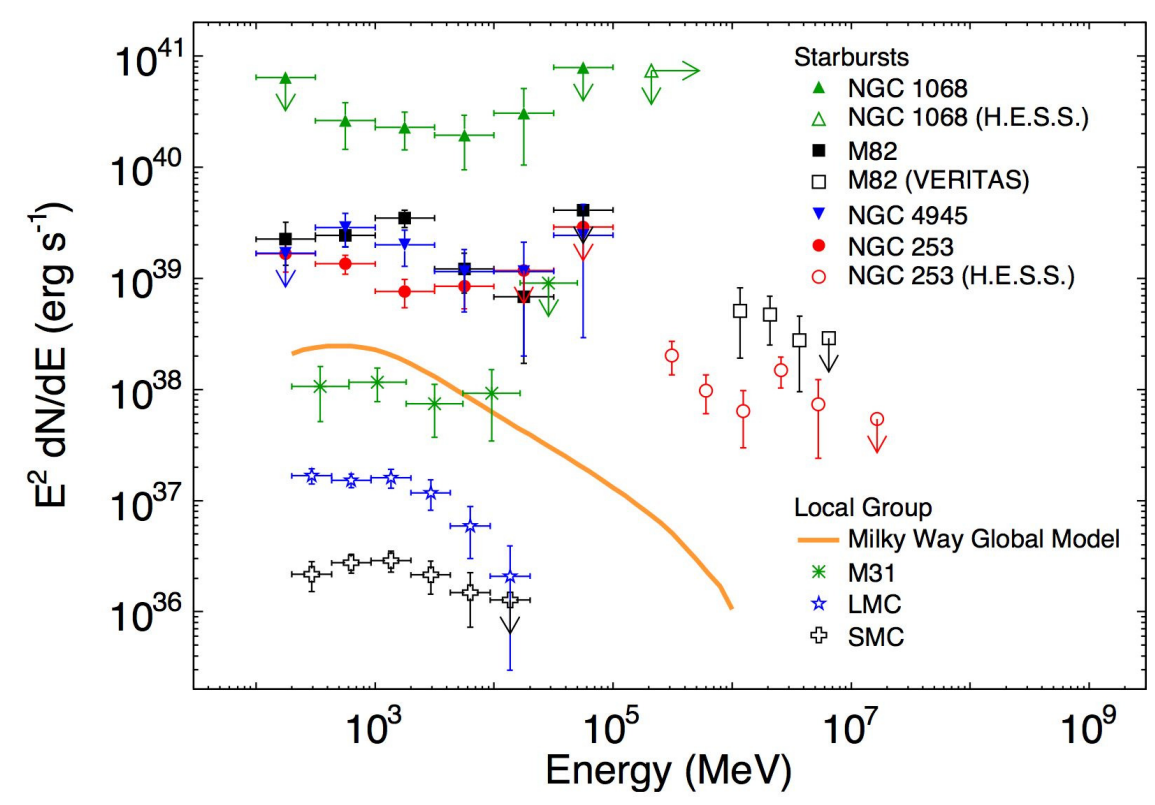

Fig. 11 Gamma-ray luminosity spectra of star-forming galaxies detected by the LAT and imaging air-Cherenkov telescopes [20].

Although star-forming galaxies are intrinsically faint sources, they are numerous in the Universe, and their combined emission may give rise to a diffuse background of cosmic gamma-ray radiation that carries an imprint of the past star formation history [104]. Following the discovery of $\mathrm{GeV}$ and $\mathrm{TeV}$ emission from starburst galaxies, the possible contribution of star-forming galaxies to the diffuse background radiation has attracted increasing attention. Estimates based on the resolved population of blazars observed by Fermi-LAT suggest that less than $30 \%$ of the diffuse extragalactic background originates from blazars [105], making a perceptible contribution from starburst galaxies to the diffuse gamma-ray background plausible. By combining the relationship between gamma-ray luminosity and SFR with an estimate of the SFR evolution with redshift derived from non-AGN galaxies [106], [20] estimate that star-forming galaxies contribute to about $\sim 10 \%$ to the diffuse gammaray background, with the bulk of the emission arising from galaxies with redshifts $z<1.5$. This estimate is still too low to explain the extragalactic gamma-ray background, suggesting that important aspects of these populations are not included in current models and/or that other high-energy source classes or diffuse processes contribute a significant fraction of the observed gamma-ray intensity. 


\section{Conclusions and outlook}

With the launch of the Fermi satellite and the advent of the modern air-Cherenkov telescope systems H.E.S.S. and VERITAS, star-forming galaxies have become a new class of gamma-ray sources that extend the study of cosmic-ray physics from the Milky Way to external galaxies. So far, 8 star-forming galaxies have been observed at $\mathrm{GeV}$ energies (SMC, LMC, Milky Way, M 31, M 82, NGC 253, NGC 1068, and NGC 4945) while 2 have been seen at TeV energies (M 82, NGC 253). The galaxies detected at TeV energies have amongst the highest SFRs in the sample, suggesting that gamma-ray spectra harden with increasing SFR.

The gamma-ray observations have confirmed the general ideas about cosmicray acceleration in galaxies, and a clear correlation between star formation activity and gamma-ray luminosity has been established. However, with the first spatiallyresolved gamma-ray images that are now available for the LMC and SMC, new challenges arise. Explaining the emission morphology of the LMC, and in particular, explaining the absence of gamma-ray emission from particularly dense regions of the interstellar medium may possibly require some revision of our ideas about cosmic-ray diffusion and penetration in dense clouds. Point source contributions may also be more important than previously thought, and cannot be neglected when studying the gamma-ray emission of galaxies, in particular if they are not spatially resolved.

The continuing observations of the Fermi satellite will probably provide more detections of star-forming galaxies in the near future. More data will also allow refining the gamma-ray spectra of the brighter systems, providing more stringent constraints on the cosmic-ray spectra in the galaxies. The freshly installed H.E.S.S. II telescope will close the gap between the $\mathrm{GeV}$ and $\mathrm{TeV}$ energy domains, and in the recent future, an unprecedented spectrum of NGC 253 promises an improved understanding of the cosmic-ray physics in that galaxy.

The new generation Cherenkov Telescope Array CTA will provide a further significant step forward. CTA will measure the diffuse gamma-ray emission surrounding nearby $(\sim 1 \mathrm{kpc})$ and powerful (a few $10^{50} \mathrm{erg}$ ) sources of cosmic rays in the Milky Way. Massive molecular clouds located in the vicinity of cosmic-ray sources will be detectable even to larger distances, comparable with the distance of the Galactic centre [107]. The detection of the overall diffuse galactic emission due to the cosmic-ray sea in the Milky Way will be challenging, but with a sufficient accumulation of observing time, advances are also expected in this area. Beyond our own Galaxy, CTA will provide unprecedented spectra of starburst galaxies, and further objects may be detected, such as the ULIRG Arp 220, providing insights into the cosmic-ray physics of the most extreme environments in the Universe.

\section{References}

1. Hess, V. F., Physik. Zeit., 12, 1084 
2. Ferrière, K. M. 2001, Rev. Mod. Phys., 73, 1031

3. Ferrière, K. M. 1998, ApJ, 497, 759

4. Ptuskin, V. S., Moskalenko, I. V., Jones, F. C., Strong, A. W., \& Zirakashvili, V. N. 2006, ApJ, 642, 902

5. Hanasz, M., Kowal, G., Otmianowska-Mazur, K., \& Lesch, H. 2004, ApJ, 605, L33

6. Hanasz, M., Otmianowska-Mazur, K., Kowal, G., \& Lesch, H. 2009, A\&A, 498, 335

7. Indriolo, N., Fields, B. D., \& McCall, B. J. 2009, ApJ, 694, 257

8. Ginzburg, V. L., \& Syrovatskii, S. I. 1964, The Origin of Cosmic Rays

9. Ellison, D. C., Drury, L. O'C., Meyer, J.-P. 1997, ApJ, 487, 197

10. Koyama, K., Petre, R., Gotthelf, E. V., et al. 1995, Nature, 378, 255

11. Aharonian, F., Bykov, A., Parizot, E., Ptuskin, V., \& Watson, A. 2012, Space Science Reviews, 166, 97

12. Cesarsky, C. J., \& Montmerle, T. 1983, Space Science Reviews, 36, 173

13. Fang, K., Kotera, K., Olinto, A. V. 2012, ApJ, 750, 118

14. Strong, A. W., Moskalenko, I. V., \& Reimer, O. 2000, ApJ, 537, 763

15. Strong, A. W., Bennett, K., Bloemen, H., et al. 1996, A\&AS, 120C, 381

16. Strong, A. W., Diehl, R., Halloin, H., et al. 2005, A\&A, 444, 495

17. Ackermann, M., Ajello, M. Atwoord, W. B., et al. 2012, ApJ, 750, 1

18. Nolan, P. L., Abdo, A. A., Ackermann, M., et al. 2012, ApJS, 199, 31

19. Ackermann, M., Ajello, M., Allafort, A., et al. 2011, Ap7, 743, 171

20. Ackermann, M., Ajello, M., Allafort, A., et al. 2012, ApJ, accepted (arXiv:1206.1346)

21. Abdo, A. A., Ackermann, M., Ajello, M., et al. 2010, A\&A, 512, 7

22. Abdo, A. A., Ackermann, M., Ajello, M., et al. 2010, A\&A, 523, 2

23. Feenberg, E., \& Primakoff, H. 1948, Phys. Rev., 73, 449

24. Hayakawa, S. 1952, Prog. Theor. Phys., 8, 571

25. Hutchinson, G. W. 1952, Philos. Mag., 43, 847

26. Morrison, P. 1958, Il Nuovo Cimento, 7, 858

27. Kraushaar, W. L., \& Clark, G. W. 1962, Phys. Rev. Lett., 8, 106

28. Clark, G. W., Garmire, G. P., \& Kraushaar, W. L. 1968, ApJ, 153, 203

29. Kraushaar, W. L., Clark, G. W., Garmire, G. P., et al. 1972, ApJ, 177, 341

30. Strong, A.W., Wdowczyk, J., \& Wolfendale, A. W. 1973, ICRC, 1, 463

31. Bignami, G. F., Fichtel, C. E., Kniffen, D. A., \& Thompson, D. J. 1975, ApJ, 199, 54

32. Dodds, D., Strong, A. W., \& Wolfendale, A. W. 1975, MNRAS, 171, 569

33. Fichtel, C. E., Simpson, G. A., \& Thompson, D. J. 1978, ApJ, 222, 833

34. Strong, A. W., Bignami, G. F., Bloemen, J. B. G. M., et al. 1982, A\&A, 115, 404

35. Dame, T. M., Ungerechts, H., Cohen, R. S., et al. 1987, ApJ, 322, 706

36. Lebrun, F., Bennett, K., Bignami, G. F., et al. 1983, ApJ, 274, 231

37. Bloemen, J. B. G. M., Bennett, K., Bignami, G. F., et al. 1984, A\&A, 135, 12

38. Bloemen, J. B. G. M., Strong, A. W., Mayer-Hasselwander, H. A., et al. 1986, A\&A, 154, 25

39. Strong, A. W., Bloemen, J. B. G. M., Dame, T. M., et al. 1988, A\&A, 207, 1

40. Hunter, S.D., Digel, S. W., De Geus, E. J., \& Kanbach, G. 1994, ApJ, 436, 216

41. Digel, S. W., Hunter, S.D., \& Mukherjee, R. 1995, ApJ, 441, 270

42. Digel, S. W., Grenier, I., Heithausen, A., Hunter, S.D., \& Thaddeus, P. 1996, ApJ, 463, 609

43. Hunter, S. D., Bertsch, D. L., Catelli, J. R., et al. 1997, ApJ, 481, 205

44. Bertsch, D. L., Dame, T. M., Fichtel, C. E., et al. 1993, ApJ, 416, 587

45. Strong, A. W., Moskalenko, I. V., \& Reimer, O. 2004, ApJ, 613, 962

46. Grenier, I. A., Casandjian, J.-M., \& Terrier, R. 2005, Science, 307, 1292

47. Sreekumar, P., Bertsch, D. L., Dingus, B. L., et al. 1992, ApJ, 400, L67

48. Fichtel, C. E., Özel, M. E., Stone, R. G., \& Sreekumar, P. 1991, ApJ, 374, 134

49. Sreekumar, P., Bertsch, D. L., Dingus, B. L., et al. 1993, Phys. Rev. Lett., 70, 127

50. Sreekumar, P., Bertsch, D. L., Dingus, B. L., et al. 1994, ApJ, 426, 105

51. Hartman, R. C., Bertsch, D. L., Bloom, S. D., et al. 1999, ApJS, 123, 79

52. Cillis, A. N., Torres, D. F., \& Reimer, O. 2005, ApJ, 621, 139

53. Atwood, W. B., Abdo, A. A., Ackermann, M., et al. 2009, ApJ, 697, 1071

54. Abdo, A. A., Ackermann, M., Ajello, M., et al. 2009, Phys. Rev. Lett., 103, 251101 
55. Abdo, A. A., Ackermann, M., Ajello, M., et al. 2009, ApJ, 703, 1249

56. Abdo, A. A., Ackermann, M., Ajello, M., et al. 2010, ApJ, 710, 133

57. Ackermann, M., Ajello, M., Baldini, L., et al. 2011, ApJ, 726, 81

58. Casandjian, J., \& Grenier, I. 2009, in 2009 Fermi Symposium, arXiv:0912.3478

59. Haslam, C. G. T., Salter, C. J., Stoffel, H., \& Wilson, W. E. 1982, A\&AS, 47, 1

60. Dobler, G., Finkbeiner, D. P., Cholis, I., Slatyer, T., \& Weiner, N. 2010, ApJ, 717, 825

61. Su, M., Slatyer, T. R., \& Finkbeiner, D. P. 2010, ApJ, 724, 1044

62. Finkbeiner, D. P. 2004, ApJ, 614, 186

63. Cheng, K.-S., Chernyshov, D. O., Dogiel, V. A., Ko, C.-M., Ip., W.-H. 2011, ApJ, 731, 17

64. Zubovas, K., \& Nayakshin, S. 2012, MNRAS, 424, 666

65. Crocker, R. M., \& Aharonian, F. 2011, Phys. Rev. Lett., 106, 101102

66. Malyshev, D., Cholis, I., \& Gelfand, J. D. 2010, 722, 1939

67. Dobler, G., Cholis, I., \& Weiner, N. 2011, ApJ, 741, 25

68. Su, M., \& Finkbeiner, D. P. 2012, ApJ, 753, 61

69. Weniger, C. 2012, arXiv:1204.2797

70. Ackermann, M., Ajello, M., Allafort, A., et al. 2012, ApJ, in press (arXiv:1207.0616)

71. Ackermann, M., Ajello, M., Allafort, A., et al. 2012, A\&A, 538, 71

72. Ackermann, M., Ajello, M., Allafort, A., et al. 2011, Science, 334, 1103

73. Montmerle, T. 1979, ApJ, 231, 95

74. Abdo, A. A., Allen, B., Aune, T., et al. 2008, ApJ, 688, 1078

75. Martin, P., Knödlseder, J., Meynet, G., \& Diehl, R. 2010, A\&A, 511, A86

76. Matsunaga, N., Feast, M. W., \& Menzies, J. W. 2009, MNRAS, 397, 933

77. Pietrzynski, G., Thompson, I. B., Graczyk, D., et al. 2009, ApJ, 697, 862

78. Kim, S., Staveley-Smith, L., Dopita, M. A., et al. 1998, ApJ, 503, 674

79. Van der Marel, R. P. 2006, in The Local Group as an Astrophysical Laboratory, ed. M. Livio, $\&$ T. M. Brown (Cambridge University Press), 47

80. Aharonian, F. 2004, in 22nd Texas Symposium on Relativistic Astrophysics, ed. by Pisin Chen, Elliott Bloom, Greg Madejski, Vahe Patrosian, p. 43

81. Pineda, J. L., Ott, J., Klein, U., et al. 2009, ApJ, 703, 736

82. Knödlseder, J. 2000, A\&A, 360, 539

83. Brandl, B. R. 2005, in Starbursts: From 30 Doradus to Lyman Break Galaxies, ed. by R. de Grijs and R. M. González Delgado. Astrophysics \& Space Science Library, Vol. 329. Dordrecht: Springer, 2005, p.49

84. Chu, Y.-H., \& Kennicutt, R. C. Jr. 1994, ApJ, 720, 737

85. Nolan, P. L., Abdo, A. A., Ackermann, M., et al. 2012, ApJS, 199, 31

86. A. A. Abdo, M. Ackermann, M. Ajello, M., et al. 2010, ApJ, 708, 1254

87. Komin, N., Djannati-Atai, A., Gallant, Y., et al. 2011, in 32nd International Cosmic Ray Conference, arXiv:1201.0639v1

88. Abdo, A. A., Ackermann, M., Ajello, M., et al. 2010, A\&A, 523, A46

89. Lenain, J.-P., \& Walter, R. 2011, A\&A, 535, 19

90. Abdo, A. A., Ackermann, M., Ajello, M., et al. 2010, ApJL, 709, L152

91. Lenain, J.-P., Ricci, C., Trler, M., Dorner, D., et al. Walter, R. 2010, A\&A, 524, A72

92. Acciari, V. A., Aliu, E., Arlen, T., et al. 2009, Nature, 462, 770

93. Acero, F., Aharonian, F., Akhperjanian, A. G., et al. 2009, Science, 326, 1080

94. Domingo-Santamar'a E., \& Torres, D. F. 2005, A\&A, 444, 403

95. Persic, M., Rephaeli, Y., \& Arieli, Y. 2008, A\&A, 486, 143

96. de Cea del Pozo, E., Torres, D. F., \& Rodriguez Marrero, A. Y. 2009, ApJ, 698, 1054

97. Lacki, B. C., Thompson, T. A., Quataert, E. 2010, ApJ, 717, 1

98. Rephaeli, Y., Arieli, Y., \& Persic, M. 2010, MNRAS, 401, 423

99. Pohl, M. 1994, A\&A, 287, 453

100. Thompson, T. A., Quataert, E., \& Waxman, E., 2007, ApJ, 654, 219

101. Lacki, B. C., Thompson, T. A., Quataert, E., Loeb, A., \& Waxman, E. 2011, ApJ, 734, 107

102. Mannheim, K., Elsässer, D., Tibolla, O. 2012, APh, 35, 797

103. Yun, M. S., Reddy, N. A., \& Condon, J. J. 2001, ApJ, 554, 803

104. Pavlidou, V., \& Fields, B. D. 2002, ApJ, 575, L5

105. Abdo, A. A., Ackermann, M., Ajello, M., et al. 2010, ApJ, 720, 435

106. Rodighiero, G., Vaccari, M., Franceschini, A., et al. 2010, A\&A, 515, A8

107. Acero, F., Bamba, A., Casanova, S., et al. 2012, APh, in press 